\title{
Bilateral Spontaneous Hyphemas in a Patient with Aplastic Anemia
}

\author{
Mohammad Z. Siddiqui ${ }^{b} \quad$ Grant Gebhard $^{a} \quad$ Alice Behrens $^{a}$ \\ Ahmed Sallam $^{\text {a }}$ Sami Uwaydat ${ }^{a}$ \\ aJones Eye Institute, Department of Ophthalmology, University of Arkansas for Medical \\ Sciences, Little Rock, AR, USA; ${ }^{b}$ College of Medicine, Department of Ophthalmology, \\ University of Arkansas for Medical Sciences, Little Rock, AR, USA
}

\section{Keywords}

Hyphema · Aplastic anemia $\cdot$ Pancytopenia $\cdot$ Anemia

\begin{abstract}
Bilateral spontaneous hyphemas are a rare ophthalmic event. Aplastic anemia is a hematologic condition with well-documented manifestations in the posterior segment but not the anterior segment. We present a patient with aplastic anemia without obvious risk factors for hyphema who developed bilateral spontaneous hyphemas. To our knowledge, this is the first reported case of bilateral spontaneous hyphemas in a patient with aplastic anemia.
\end{abstract}

\section{(C) 2018 The Author(s)}

Published by S. Karger AG, Basel

\section{Introduction}

Aplastic anemia (AA) is a rare and serious disease of bone marrow failure, which has an incidence of 2 per million in Western countries and 4-6 per million in Asia [1, 2]. It is caused by the suppression of hematopoietic stem cells within the bone marrow, with resulting pancytopenia. It has been associated with multiple pathologic processes, including viral infections, autoimmune mechanisms, and direct insult to hematopoietic stem cells by drugs, chemicals, or irradiation; however, the majority of cases are idiopathic [3]. Posterior segment complications in the setting of AA have been well documented. They include retinal hemorrhages 
(67\%), vitreous or subhyaloid hemorrhages (13\%), peripheral retinopathy (5.5\%), cotton wool spots (4\%), and optic disc edema (4\%) $[2,4]$. Anterior segment findings included subconjunctival hemorrhages, Sjögren's syndrome (4\%), anterior segment ischemia (0.5\%), and scleral necrosis $(0.5 \%)$ [2]. Although posterior segment hemorrhage is a known clinical presentation in patients with AA, hyphema has not been reported in association with AA.

We present a case of bilateral spontaneous hyphemas in a patient with refractory AA but with no prior ocular history or surgery, systemic use of anticoagulation or antiplatelet agents, trauma, or other hematological disorders.

\section{Case Report}

A 43-year-old African American female with refractory AA and end stage renal disease was admitted to the hospital for malfunction of hemodialysis graft. Past medical history included superior vena cava syndrome, bilateral subclavian to atrial bypass surgery, and type 2 diabetes mellitus. Her hospital course was complicated by esophageal bleeding and pneumonia. Her AA was treated with a standard combination of antithymocyte globulin and cyclosporine. On hospital day 54, she reported an acute and painless fogging of the vision of the left eye. Her uncorrected near visual acuity was $20 / 25$ in the right eye and 20/20 in the left eye. Intraocular pressures were $19 \mathrm{~mm} \mathrm{Hg}$ in both eyes. Slit-lamp examination revealed a phakic patient with normal anterior segment in the right eye and a 2-mm layered hyphema of the left eye. Dilated fundus exam revealed dilated retinal veins and intraretinal hemorrhages in both eyes and a subhyaloid hemorrhage in the left eye. Her hemoglobin was $6.2 \mathrm{~g} / \mathrm{dL}$, and platelet count was $2,000 / \mu \mathrm{L}$.

The next day, the patient reported decreased vision in the right eye. Visual acuity had worsened to count fingers at 1 foot in the right eye. Intraocular pressures were $19 \mathrm{~mm} \mathrm{Hg}$ in both eyes. Slit-lamp examination revealed a 3-mm hyphema in the right eye and a resolving 1-mm hyphema in the left eye (Fig. 1). Gonioscopy revealed open angles with no iris neovascularization in either eye. Funduscopic exam was stable in both eyes compared to the previous day (Fig. 2). Fluorescein angiography showed normal retinal vasculature filling in both eyes, with no areas of ischemia (Fig. 3). Macular optical coherence tomography showed normal retinal layers. The patient was started on topical prednisolone acetate in both eyes and atropine in the right eye. The intraocular pressure remained stable, and the vision slowly improved. The hyphemas resolved in both eyes 6 days after initial presentation.

\section{Discussion/Conclusion}

Spontaneous, nontraumatic, bilateral hyphema is rarely reported. Spontaneous hyphemas are sometimes associated with juvenile xanthogranuloma, warfarin use, or congenital vascular anomalies of the iris, such as microhemangiomas, vascular tufts, or persistent vascularized pupillary membranes [5-8]. Hematologic abnormalities previously reported with bilateral spontaneous hyphemas are neonatal thrombocytopenia and idiopathic thrombocytopenic purpura $[9,10]$. Several case series have examined the ocular complications of AA $[3,4]$. As previously mentioned, posterior segment complications include retinal hemorrhages, vitreous or subhyaloid hemorrhages, peripheral retinopathy, cotton wool spots, and optic disc edema [2]. Anterior segment involvement may lead to subconjunctival hemorrhages, 
Sjögren's syndrome, anterior segment ischemia, and scleral necrosis [2]. To our knowledge, there are no reported cases of spontaneous hyphemas associated with AA.

Chronic anemia is believed to be one of the leading factors for posterior segment bleeding in patients with AA [2]. Dilation of the retinal venous system can occur in order to compensate for low oxygen carrying capacity. This places the endothelium of blood vessels under stress and leads to intraocular hemorrhage. In AA, megakaryocytopoiesis is impaired due to decreased numbers of hematopoietic stem cells, which leads to diminished mature platelet production and subsequent thrombocytopenia [1]. Systemic bleeding is typically not seen until the platelet count is below $10,000-20,000 / \mu \mathrm{L}$. A synergistic effect between anemia and thrombocytopenia has been demonstrated, with a higher rate of retinal bleeding seen in patients where both are present [11].

In our patient, no vascular tuft, pupillary membrane, microhemangioma of the iris, or neovascularization of the anterior segment or angle was seen. Although there were hemorrhages in the posterior segment as expected with her severe anemia and thrombocytopenia, there was no neovascularization or vascular occlusion on fluorescein angiography. The bilateral venous tortuosity in the absence of venous dilation or retinal ischemia was likely due to congenital venous tortuosity.

There were no other contributing risk factors or exam findings in this patient that could explain the spontaneous hyphema. We believe that the severity and refractory nature of her AA was the most likely cause of the bilateral spontaneous hyphemas, given the absence of other contributing anatomic risk factors. Throughout her hospital stay, our patient was thrombocytopenic with platelet count remaining between 3,000-27,000/ $\mu \mathrm{L}$, even after resolution of the hyphema. The hyphemas resolved in both eyes without sequelae.

We recommend hematologic workup and appropriate referral for evaluation of anemia and thrombocytopenia for patients with new-onset spontaneous hyphema without trauma, iris vascular abnormality, or neovascularization of the anterior segment and posterior segment.

\section{Acknowledgement}

We acknowledge Jones Eye Institute, University of Arkansas for Medical Sciences College of Medicine.

\section{Statement of Ethics}

The authors have no ethical conflicts to disclose.

\section{Disclosure Statement}

The authors have no conflicts of interest to declare.

\section{Funding Sources}

The authors did not receive funding. 


\section{Case Reports in Ophthalmology} \begin{tabular}{l|l}
\hline DOI: $10.1159 / 000493784$ & $\begin{array}{l}\text { (c) } 2018 \text { The Author(s). Published by S. Karger AG, Basel } \\
\text { www.karger.com/cop }\end{array}$
\end{tabular}

Siddiqui et al.: Bilateral Spontaneous Hyphemas in a Patient with Aplastic Anemia

\section{Author Contributions}

Mohammad Zia Siddiqui wrote and edited the manuscript. Dr. Grant Gebhard examined the patient, wrote the manuscript, and edited the manuscript. Dr. Alice Behrens examined the patient, wrote and edited the manuscript. Dr. Ahmed Sallam wrote and edited the manuscript. Dr. Sami Uwaydat is the attending physician for this patient, and he examined the patient, wrote and edited the manuscript.

\section{References}

1 Incidence of aplastic anemia: the relevance of diagnostic criteria. By the International Agranulocytosis and Aplastic Anemia Study. Blood. 1987 Dec;70(6):1718-21.

2 Mansour AM, Lee JW, Yahng SA, Kim KS, Shahin M, Hamerschlak N, et al. Ocular manifestations of idiopathic aplastic anemia: retrospective study and literature review. Clin Ophthalmol. 2014 Apr;8:777-87.

3 Kaufman DW, Kelly JP, Levy M, Shapiro S. The drug etiology of agranulocytosis and aplastic anemia. New York, NY: Oxford; 1991.

4 Mansour AM, Salti HI, Han DP, Khoury A, Friedman SM, Salem Z, et al. Ocular findings in aplastic anemia. Ophthalmologica. 2000;214(6):399-402.

5 Nunziata BR. Idiopathic bilateral spontaneous hyphemas. Ann Ophthalmol. 1990 Dec;22(12):472-4.

6 Perry HD, Mallen FJ, Sussman W. Microhaemangiomas of the iris with spontaneous hyphaema and acute glaucoma. Br J Ophthalmol. 1977 Feb;61(2):114-6.

7 Strauss EC, Aldave AJ, Spencer WH, Branco BC, Barsness DA, Calman AF, et al. Management of prominent iris vascular tufts causing recurrent spontaneous hyphema. Cornea. 2005 Mar;24(2):224-6.

8 Laidlaw DA, Bloom PA. Spontaneous hyphaema arising from a strand of persistent pupillary membrane. Eur J Ophthalmol. 1993 Apr-Jun;3(2):98-100.

9 Jerónimo M, Azenha C, Mesquita J, Pereira DF. A rare manifestation of neonatal alloimmune thrombocytopaenia. BMJ Case Rep. 2014 Jun 2. doi: 10.1136/bcr-2014-204393.

10 McDonald CJ, Raafat A, Mills MJ, Rumble JA. Medical and surgical management of spontaneous hyphaema secondary to immune thrombocytopenia. Br J Ophthalmol. 1989 Nov;73(11):922-5.

11 Carraro MC, Rossetti L, Gerli GC. Prevalence of retinopathy in patients with anemia or thrombocytopenia. Eur J Haematol. 2001 Oct;67(4):238-44.
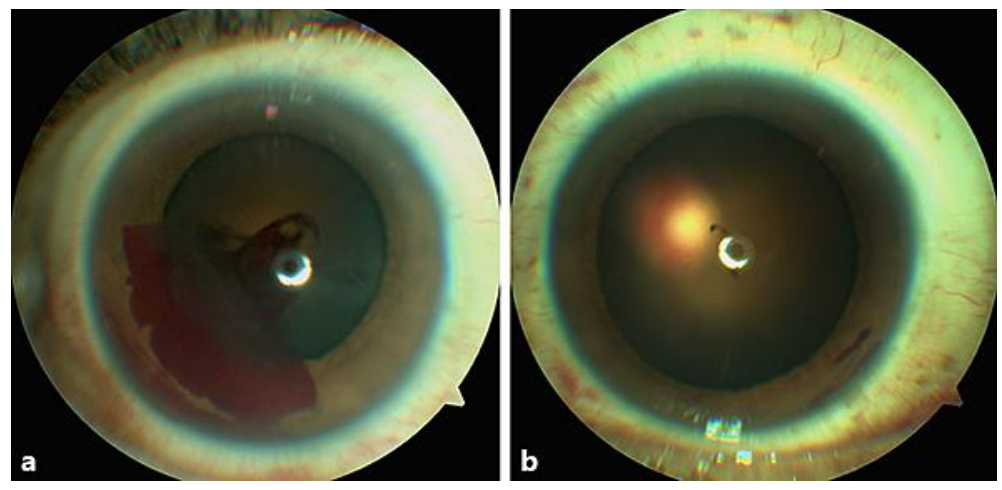

Fig. 1. Anterior segment photograph of the right eye showing hyphema (a) and of the left eye showing resolving hyphema (b). 


\section{Case Reports in Ophthalmology}
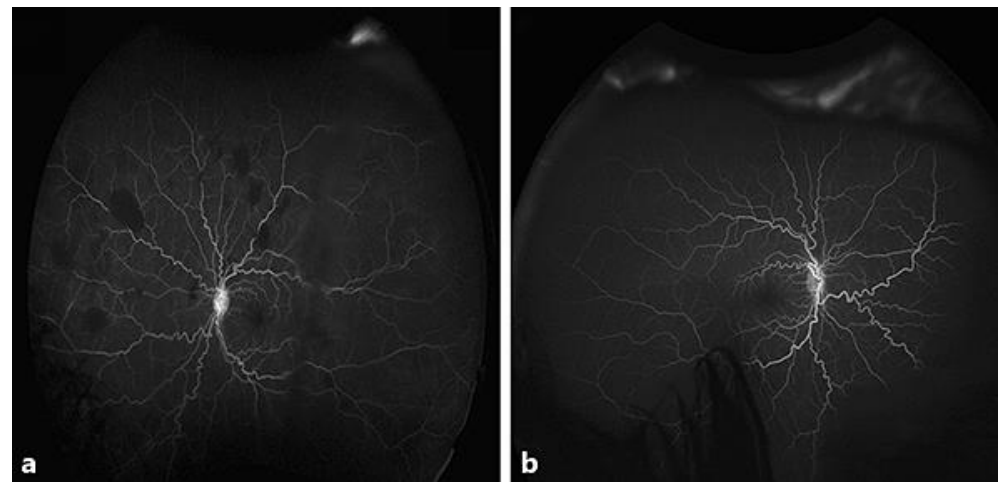

Fig. 2. a Mid-phase fluorescein angiography of the right eye showing normal filling without leakage. b Midphase fluorescein angiography of the left eye showing normal filling and multiple intraretinal hemorrhages.
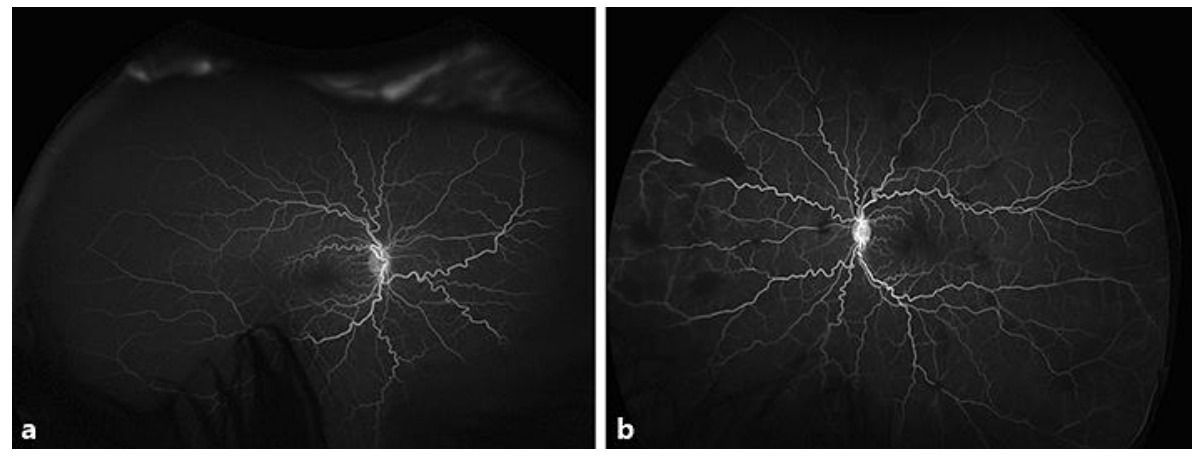

Fig. 3. a Fundus photograph of the right eye showing a few peripheral intraretinal hemorrhages. $\mathbf{b}$ Fundus photograph of the left eye showing multiple intraretinal hemorrhages. 